\title{
Particle-based modeling and meshless simulation of flows with smoothed particle hydrodynamics
}

\author{
Sofos F., Liakopoulos A.*, Karakasidis T.E. \\ Hydromechanics and Environmental Engineering Laboratory, Department of Civil Engineering, University of Thessaly, Pedion Areos, \\ GR-38334, Volos, Greece \\ Received: 31/01/2019, Accepted: 06/05/2019, Available online: 05/06/2019 \\ *to whom all correspondence should be addressed: e-mail: aliakop@civ.uth.gr \\ https://doi.org/10.30955/gnj.003052
}

\begin{abstract}
Smoothed Particle Hydrodynamics (SPH) is a promising simulation technique in the family of Lagrangian mesh-free methods, especially for flows that undergo large deformations. Particle methods do not require a mesh (grid) for their implementation, in contrast to conventional Computational Fluid Dynamics (CFD) methods. Conventional CFD algorithms have reached a very good level of maturity and the limits of their applicability are now fairly well understood. In this paper we investigate the application of the SPH method in Poiseuille and transient Couette flow along with a free surface flow example. Algorithmically, the method is viewed within the framework of an atomic-scale method, Molecular Dynamics (MD). In this way, we make use of $\mathrm{MD}$ codes and computational tools for macroscale systems.
\end{abstract}

Keywords: Smoothed particle hydrodynamics (SPH), molecular dynamics, Poiseuille flow, Couette flow, tank drainage.

\section{Introduction}

Lagrangian methods are based on the concept of describing fluid flows by following the motion of fluid particles. This appears to overcome numerical difficulties associated with large deformations. In this work we discuss the common concepts and similarities underlying three particle methods: Molecular Dynamics (MD), Dissipative Particle Dynamics (DPD) and Smooth Particle Hydrodynamics (SPH). These methods are appropriate for different time and length scales.

In an MD simulation, interactions between particles (atoms or molecules) are calculated and this seems to be the most appropriate method to study flows at the nanoscale since the assumption of continuous medium, conventionally employed in fluid dynamics, cannot be applied. At these very small scales, a wide range of studies have shown that as channel dimensions decrease, solid wall particles interact with the fluid and control its behavior, such as fluid atom positions, velocity, temperature and transport properties such as diffusion coefficient, shear viscosity and thermal conductivity (Spetsiotis et al., 2018; Spareboom et al., 2009; Sofos et al., 2009; Eral et al., 2009). On a larger time and/or spatial scale MD simulations become very time consuming and need large computational resources. At these scales, DPD is considered as a simulation method that bridges the gap between atomistic and mesoscopic simulation and has been successfully applied in modeling complex fluids in periodic domains (Pivkin and Karniadakis, 2005; Lei et al., 2008; Li et al., 2015).

As far as macroscale methods are concerned, the central idea in SPH is the subdivision of the fluid system under study to a number of moving particles ("chunks" or blobs of matter) (Monaghan, 1988, 1992). The conservation laws of continuum fluid dynamics, in the form of partial differential equations, are transformed into their particle forms by integral equations through the use of an interpolation function that gives the kernel estimate of the field variables at a point. Information is extracted only at discrete points (the particles) and the integrals are evaluated as sums over neighboring particles. Each fluid particle has a constant mass and time-dependent velocity, density, pressure, dynamic viscosity, temperature (as needed). In the SPH framework the governing PDEs describing the system in motion are transformed to a number of ordinary differential equations (ODEs). For example, a possible form of SPH formulation of conservation of momentum and mass PDEs leads to a set of ODEs for the velocities and densities of the particles which can be integrated by a numerical method of integration of ODEs (e.g. Verlet, Euler, Runge-Kutta, etc). The positions of the particles are then calculated by integrating the velocity. Detailed work on SPH can be found in (Gingold and Monaghan, 1977; Koumoutsakos, 2005; Shao, 2009; Bouscasse et al., 2013; Bian et al., 2015).

$\mathrm{SPH}$ is well suited for flows of liquids with free surface such as wave propagation, wave/structure interaction, sloshing to mention a few. Furthermore, SPH is very useful in applications in soil mechanics and water resources engineering such as: flood wave propagation modeling, 
floodplain inundation predictions, open channel hydraulics. Environmental applications at small scales include sensors for air and water quality, pathogen detection devices and biological security threat mitigation.

The ideas of SPH have "merged" with the mesoscopic method DPD (Muller et al., 2015). The result is a method frequently referred to as SDPD (for smooth DPD). In SDPD the discretization of the Navier-Stokes equation follows the methodology of SPH but, in addition the method includes the thermal fluctuations as in the original DPD method. SDPD is appropriate for simple, complex and biological fluids. It is also proposed to develop strategies/techniques for coupling these methods across scales (nano, meso, macro) using the conceptual framework of peridynamics (i.e. non-local theories of mechanics) as can be found in Silling (2010).

We applied the SPH simulation method on a software platform that has been widely used for research, primarily for Molecular Dynamics simulations of atomistic systems, LAMMPS (Large-scale Atomic/Molecular Massively Parallel Simulator, see Plimpton (1995)), based on a previous work by Liakopoulos et al. (2018). Due to its particle nature, SPH is directly compatible with the existing code architecture and data structures present in LAMMPS for MD (Sofos et al., 2009, 2013; Liakopoulos et al., 2016). Furthermore, its parallel nature offers a boost in all simulations that could be executed in parallel tasks (Herault et al., 2010). It would be interesting to develop reliable models of length scale coupling for problems with multiple physics and multiple scales, such as ink-jet printing, DNA and protein micro-/nano-arraying, and fabrication of particles and capsules for controlled release of medicines (Liu and Liu, 2010). Towards these directions, in this work we focus on reproducing CFD examples with SPH and investigate three SPH test cases, the development of transient Couette flow, Poiseuille flow, and a water tank drainage example.

\section{Smoothed particle hydrodynamics formulation}

\subsection{SPH mathematics}

In this section we present the basic steps in developing an SPH formulation for a given partial differential equation, while referring to the case of Navier-Stokes equations and the energy equation for a Newtonian fluid in parallel. A detailed description of the mathematics of the method can be found in Liakopoulos et al. (2018).

The starting point of an SPH formulation is an integral approximation of a function $f(\mathbf{x})$. In its ideal form the approximation has the form of the identity

$$
f(\mathbf{x})=\int_{\Omega} f\left(\mathbf{x}^{\prime}\right) \delta\left(\mathbf{x}-\mathbf{x}^{\prime}\right) \mathrm{d} \mathbf{x}^{\prime}
$$

where $\mathbf{x}$ is the position vector and $\delta\left(\mathbf{x}-\mathbf{x}^{\prime}\right)$ is the Dirac's delta function defined as

$\delta\left(\mathbf{x}-\mathbf{x}^{\prime}\right)=\left\{\begin{array}{lll}1 & \text { when } & \mathbf{x}=\mathbf{x}^{\prime} \\ 0 & \text { when } & \mathbf{x} \neq \mathbf{x}^{\prime}\end{array}\right.$

If we replace the delta function by a kernel of the integral approximation, i.e., $\mathbf{W}\left(\mathbf{x}-\mathbf{x}^{\prime} ; \mathrm{h}\right)$, this function plays the role of a smoothing function over a spatial neighbourhood of dimension $\mathrm{h}$.

$f(\mathbf{x}) \approx \int_{\Omega} f\left(\mathbf{x}^{\prime}\right) \mathrm{W}\left(\mathbf{x}-\mathbf{x}^{\prime} ; \mathrm{h}\right) \mathrm{d} \mathbf{x}^{\prime}$

The choice of the kernel is important for the success of the method.

Next, the flow domain is discretized by a set of point masses (fluid particles). The smoothing kernels (also known as interpolation kernels) are centered at the point masses. For a variable $f(\mathbf{x})$, we calculate its value at $\mathbf{x}_{i}$ by

$$
f\left(\mathbf{x}_{i}\right)=\sum_{j=1}^{\mathrm{N}} \mathrm{m}_{j} \frac{f_{j}}{\rho_{j}} \mathrm{~W}\left(\mathbf{x}_{i}-\mathbf{x}_{j}\right)
$$

and the gradient at position $\mathbf{x}_{i}$ by

$$
\nabla f\left(\mathbf{x}_{i}\right)=\nabla \sum_{j=1}^{\mathrm{N}} \mathrm{m}_{j} \frac{f_{j}}{\rho_{j}} \mathrm{~W}_{i j}=\sum_{j=1}^{\mathrm{N}} \mathrm{m}_{j} \frac{f_{j}}{\rho_{j}} \nabla_{j} \mathrm{~W}_{i j}
$$

In rectangular Cartesian coordinates $\left(x^{1}, x^{2}, x^{3}\right)$ the conservation equations for mass, momentum and energy are written in indicial notation as

$$
\begin{aligned}
& \frac{\mathrm{D} \rho}{\mathrm{Dt}}=-\rho \frac{\partial v^{\beta}}{\partial x^{\beta}} \\
& \frac{\mathrm{D} v^{a}}{\mathrm{Dt}}=\frac{1}{\rho} \frac{\partial \sigma^{a \beta}}{\partial x^{\beta}}+\mathrm{b}^{a} \\
& \frac{\mathrm{De}}{\mathrm{Dt}}=\frac{1}{\rho} \sigma^{a \beta} \frac{\partial v^{a}}{\partial x^{\beta}}-\frac{\partial q^{a}}{\partial x^{a}}
\end{aligned}
$$

where $\rho$ is the fluid density, $\left(v^{1}, v^{2}, v^{3}\right)$ are the components of the velocity vector, $\sigma^{\alpha \beta}$ the components of the total stress tensor, $\left(q^{1}, q^{2}, q^{3}\right)$ is the heat flux vector, $\left(b^{1}, b^{2}, b^{3}\right)$ is the body force per unit mass, $a=1,2,3$ and $\beta=1,2,3$. Here, repeated indices imply summation from 1 to 3 . Incorporating the constitutive equations for Newtonian fluids we obtain the well known Navier-Stokes equations. Incorporating the SPH particle approximation for the dependent variables and their derivatives (Eqs. 3 and 4) we obtain the SPH equations for the Navier-Stokes, continuity and energy equations as follows:

$$
\begin{aligned}
& \frac{\mathrm{D} \rho_{i}}{\mathrm{Dt}}=\sum_{j=1}^{\mathrm{N}} \mathrm{m}_{j} v_{i j}^{\beta} \frac{\partial \mathrm{W}_{i j}}{\partial x_{i}^{\beta}} \\
& \frac{\mathrm{D} v_{i}^{a}}{\mathrm{Dt}}=-\sum_{j=1}^{\mathrm{N}} \mathrm{m}_{j}\left(\frac{\sigma_{i}^{\alpha \beta}}{\rho_{i}^{2}}+\frac{\sigma_{j}^{\alpha \beta}}{\rho_{j}^{2}}\right) \frac{\partial \mathrm{W}_{i j}}{\partial x_{i}^{\beta}}+b_{i}^{a} \\
& \frac{\mathrm{De}_{i}}{\mathrm{Dt}}=\frac{1}{2} \sum_{j=1}^{\mathrm{N}} \mathrm{m}_{j}\left(\frac{\mathrm{p}_{i}}{\rho_{i}^{2}}+\frac{\mathrm{p}_{j}}{\rho_{j}^{2}}\right) v_{i j}^{\beta} \frac{\partial \mathrm{W}_{i j}}{\partial x_{i}^{\beta}}+\frac{\mu_{i}}{2 \rho_{i}} \varepsilon_{i}^{a \beta} \varepsilon_{i}^{a \beta}
\end{aligned}
$$

where $v_{i j}=v_{i}-v_{j}, \varepsilon^{\alpha \beta}=\frac{\partial v^{\beta}}{\partial x^{\alpha}}+\frac{\partial v^{\alpha}}{\partial x^{\beta}}-\frac{2}{3}(\nabla \cdot \overrightarrow{\mathrm{V}}) \delta^{\alpha \beta}, \sigma^{\alpha \beta}=$ $-\mathrm{p} \delta^{\alpha \beta}+\tau^{\alpha \beta}$, and $\tau^{\alpha \beta}$ are the components of the stress deviator, $\mathrm{m}_{j}$ is the mass of the $j^{\text {th }}$ particle, $\rho_{i}$ is the density of the $i^{\text {th }}$ particle, $\mathrm{p}_{i}$ is the pressure of the $i^{\text {th }}$ particle, $\left(v_{i}^{1}, v_{i}^{2}, v_{i}^{3}\right)$ the velocity of the $i^{\text {th }}$ particle, and $\mu_{i}$ the dynamic viscosity coefficient of $i^{\text {th }}$ particle. 


\subsection{The choice of the smoothing function}

It is obvious that the choice of the smoothing function $\mathrm{W}$ and the smoothing length, $h$, is very important and can lead to success or failure of the method. A smoothing function must have a number of properties such as the "property of unity", compact support (i.e., local support), positivity, decay, smoothness, symmetry, as well as the "Delta function property". Among these seven desirable properties, two of them are indispensable:

$$
\int_{\Omega} W\left(\mathbf{x}-\mathbf{x}^{\prime} ; h\right) d \mathbf{x}^{\prime}=1 \quad \text { ("unity" property) }
$$

and

$$
\lim _{h \rightarrow 0} W\left(\mathbf{x}-\mathbf{x}^{\prime} ; \mathrm{h}\right)=\delta\left(\mathbf{x}-\mathbf{x}^{\prime}\right) \quad \text { (“Delta function" property) }
$$

\subsection{SPH equations as solved in LAMMPS}

In the LAMMPS implementation the field variables are $\{\rho$, $\mathbf{v}, e, P, Q\}$ that is density, velocity, internal energy, the stress tensor, and the heat flux vector. The discretized equations are:

\section{Local density for particle $i$}

$\rho_{i}=\sum_{j=1}^{\mathrm{N}} \mathrm{m}_{j} \frac{\rho_{j}}{\rho_{j}} \mathrm{~W}_{i j}=\sum_{j=1}^{\mathrm{N}} \mathrm{m}_{j} \mathrm{~W}_{i j}$

This is frequently referred in the mathematical literature as "partition of unity".

Momentum equation for particle $i$

$$
\frac{\mathrm{d} \mathbf{v}_{i}}{\mathrm{dt}}=-\frac{\mathrm{P}_{i}}{\rho_{i}^{2}} \cdot \sum_{j=1}^{\mathrm{N}} \mathrm{m}_{j} \nabla_{j} \mathrm{~W}_{i j}-\sum_{j=1}^{\mathrm{N}} \mathrm{m}_{j} \frac{\mathrm{P}_{j}}{\rho_{j}^{2}} \nabla_{j} \mathrm{~W}_{i j}
$$

where $P$ is the stress (pressure) tensor. Note that the pair-wise forces are

$\mathbf{f}_{i}=\mathrm{m}_{i} \frac{\mathrm{d} \mathbf{v}_{i}}{\mathrm{dt}}=-\sum_{j=1}^{\mathrm{N}} \mathrm{m}_{i} \mathrm{~m}_{j}\left(\frac{\mathrm{P}_{i}}{\rho_{i}^{2}}+\frac{\mathrm{P}_{j}}{\rho_{j}^{2}}\right) \nabla_{j} \mathrm{~W}_{i j}$

\section{Continuity equation}

$$
\frac{\mathrm{d} \rho_{i}}{\mathrm{dt}}=-\sum_{j=1}^{\mathrm{N}} \mathrm{m}_{j} \mathbf{v}_{j} \nabla_{j} \mathrm{~W}_{i j}-\mathbf{v}_{i} \sum_{j=1}^{\mathrm{N}} \mathrm{m}_{j} \nabla_{j} \mathrm{~W}_{i j}-\sum_{j=1}^{\mathrm{N}} \mathrm{m}_{j} \mathbf{v}_{i j} \nabla_{j} \mathrm{~W}_{i j}
$$

\section{Energy equation}

$$
\mathrm{m}_{i} \frac{\mathrm{d} e_{i}}{\mathrm{dt}}=-\frac{1}{2} \sum_{j=1}^{\mathrm{N}} \mathrm{m}_{i} \mathrm{~m}_{j}\left(\frac{\mathrm{P}_{i}}{\rho_{i}^{2}}+\frac{\mathrm{P}_{j}}{\rho_{j}^{2}}\right): \mathbf{v}_{i j} \nabla_{j} \mathrm{~W}_{i j}-\sum_{j=1}^{\mathrm{N}} \frac{\mathrm{m}_{i} \mathrm{~m}_{j}}{\rho_{i} \rho_{j}} \frac{\left(\kappa_{i}+\kappa_{j}\right)\left(\mathrm{T}_{i}-\mathrm{T}_{j}\right)}{\mathrm{r}_{i j}^{2}} \mathbf{r}_{i j} \cdot \nabla_{j} \mathrm{w}_{i j}
$$

\section{Newman-Richter type artificial viscosity}

Monaghan has introduced an artificial viscosity term in order to avoid instabilities in this SPH formulation of the $\mathrm{N}-\mathrm{S}$ equations. It is adopted in the LAMMPS formulation so that the pair-wise forces are modified and take the form

$$
\mathbf{f}_{i}=\mathrm{m}_{i} \frac{\mathrm{d} \mathbf{v}_{i}}{\mathrm{~d} t}=-\sum_{j=1}^{N} \mathrm{~m}_{i} \mathrm{~m}_{j}\left(\frac{\mathrm{P}_{i}}{\rho_{i}^{2}}+\frac{\mathrm{P}_{j}}{\rho_{j}^{2}}+\Pi_{i j}\right) \nabla_{j} \mathrm{~W}_{i j}
$$

with

$$
\Pi_{i j}=-\alpha \mathrm{h} \frac{\mathrm{c}_{i}+\mathrm{c}_{j}}{\rho_{i}+\rho_{j}} \frac{\mathbf{v}_{i j} \cdot \mathbf{r}_{i j}}{r_{i j}^{2}+\varepsilon \mathrm{h}^{2}}
$$

where $c_{i}=$ speed of sound of particle $i, c_{j}=$ speed of sound of particle $j, \alpha=$ auxiliary factor for control of dissipation, $\mathbf{r}_{i j}$ the distance vector between $i^{\text {th }}$ and $j^{\text {th }}$ particle, $\varepsilon=$ auxiliary factor used to avoid singularities when $r_{i j} \rightarrow 0$. As a rule of thump $\varepsilon \approx 0.01$. The energy equation has to be also modified.

\subsection{Some remarks about SPH formulations for fluids}

In relation to the application of the SPH method in fluid dynamical problems we should mention that the treatment of pressure for incompressible flow can be carried out either through an equation of state or by enforcing the incompressibility condition via a Poisson equation for pressure. Another important issue in viscous water flows is the treatment of viscosity, which is a key quantity in determining water transport. In addition, the computational enforcement of boundary conditions (especially inlet-outlet boundary conditions) requires further development (Lykov et al., 2015; Lei et al., 2011). Collision detection at impermeable solid boundaries is also very important.

\section{Results and discussion}

In order to estimate the effectiveness and accuracy of the SPH method in LAMMPS, we have chosen to reproduce three classical CFD and Computational Hydraulics examples, the development of a transient Couette flow, Poiseuille flow in various channel dimensions, and the well-documented water tank drainage example. Snapshots of the simulations, as well as velocity and density profiles where possible are presented in each case.

\subsection{Transient Couette flow}

A 3-D rectangular simulation box is created for unsteady Couette flow, as shown in Figure 1 . The model screenshot was created with OVITO (Stukowski, 2010). The dimensions in $x-, y-$ and $z$-directions are $\left(L_{x}, L_{y}, L_{z}\right)=\left(2 \times 10^{-2} \mathrm{~mm}, 1 \times 10^{-3} \mathrm{~m}, 1 \times 10^{-3} \mathrm{~m}\right)$. Wall and fluid particles are set on fcc (face centered cubic) sites in the beginning of the simulation and remain on their initial position until the upper rigid plate moves. The solid walls are simulated by particles which balance the pressure of inner fluid particles and prevent them from penetrating the wall. We make the distinction between wall particles and fluid particles by their ability to move or not: fluid particles move while wall particles remain in their initial positions during simulation. The upper wall is given a constant velocity, $v_{x}=1 \times 10^{-2} \mathrm{~m} / \mathrm{s}$, in the $x$-direction and drives the flow due to friction. The channel consists of 400 wall particles and 3400 water particles. The upper and lower walls are formed by two layers of wall particles. The nominal density for walls and fluid is constant $\rho=1000 \mathrm{~kg} / \mathrm{m}^{3}$. Periodic conditions are enforced in the $x$ - and $z$ - directions. The simulation runs with a timestep of $\Delta t=5 \times 10^{-5} \mathrm{~s}$. 


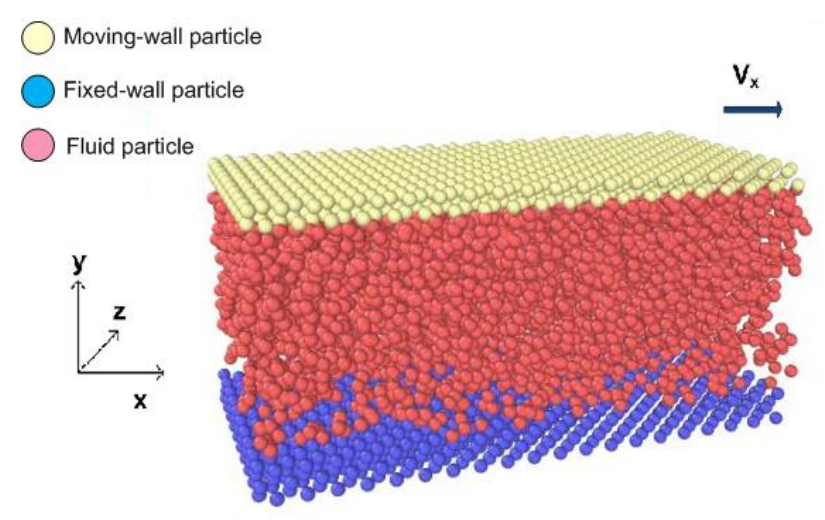

Figure 1. Couette flow model

In the beginning of a simulation, water particles are assigned random initial velocities in order to reach the desired temperature, before the upper plate moves. The system reaches equilibrium after an equilibration run of about $1 \times 10^{6}$ timesteps. Then, the upper plate is given a constant velocity and production runs start.

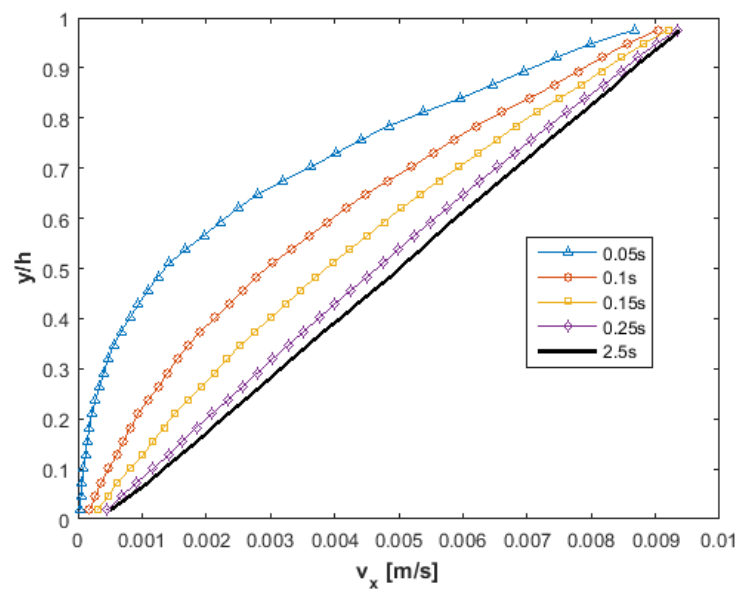

Figure 2. $x$-Velocity profile across the $y$-direction as a function of the simulation time

Figure 2 shows the resulting velocity profile across the $y$-direction extracted at various times of the simulation. As it can be seen, we obtain transient behaviour as the flow evolves in time and reaches steady state between 0.25 and $2.5 \mathrm{~s}$. At steady state, the velocity profile is linear and this result is in agreement to similar problems at these scales (Hu and Adams, 2006), although, Song et al. (2018) note that at such scale, the SPH simulation converges, but the deviation from the analytical solution is considerable. For our model, the time $t_{s}$ required to reach steady flow (asymptotically) is in agreement with continuum theory prediction (White, 1991; Liakopoulos and Sofos, 2016).

\subsection{Poiseuille flow}

The computational domain for SPH Poiseuille flow consists of a simulation box, periodic on $x$ - and $z$-dimensions, similar to the previous Couette example (Figure 1), with stationary upper wall. Flow originates due to the application of an external force $F_{\text {ext }}$ at the $x$-direction on all fluid particles, which acts as a LAMMPS analog to the application of pressure difference to induce the flow in macroscopic systems. In such systems, in LAMMPS simulations, it is common to use periodic conditions for the channel in order to simulate the flow and the flow is driven not by a pressure difference $\Delta p$ but by an external force $F_{\text {ext }}$ applied to each of the $N$ fluid particles in the model. Consequently, $N \cdot F_{\text {ext }} \leftrightarrow \Delta p \cdot A$, where $A$ is the crosssection area. Corresponding $d p / d x$ values for each value of $F_{\text {ext }}$ used in our simulations are shown in Table 1.

Table 1. Corresponding $d p / d x$ and $F_{\text {ext }}$ values

\begin{tabular}{|ccccc|}
\hline$F_{\text {ext }}\left(\times 10^{-9} \mathrm{~N}\right)$ & 1.0 & 2.5 & 5.0 & 10.0 \\
\hline$d p / d x\left(\times 10^{-3} \mathrm{~Pa} / \mathrm{m}\right)$ & -3.4 & -8.5 & -17.0 & -34.0 \\
\hline
\end{tabular}

Temperature remains constant $(T=300 \mathrm{~K})$ throughout the simulations. In the beginning of a simulation, water particles are assigned random initial velocities in order to reach the desired temperature. The system reaches equilibrium state after a run of about $1 \times 10^{6}$ timesteps, where the timestep is $\Delta t=0.01 \mathrm{~s}$. Next, an external force is applied to drive the flow. A thermostatting mechanism (a common practice in LAMMPS) is incorporated to keep system temperature constant at $300 \mathrm{~K}$, since the application of the external force adds energy to the flow and it has to be removed through the thermostatted walls.

Figure 3 presents particle number density profiles across the channel, for each magnitude of $F_{\text {ext }}$ used. Density profiles reveal density variations that develop in a fluid due to fluid structuring near the walls. The instantaneous particle number density is expressed as the number of particles located in each simulation bin at a specific timestep. This number of particles is averaged over the total simulation time and a time-averaged value for each bin is extracted and shown here.

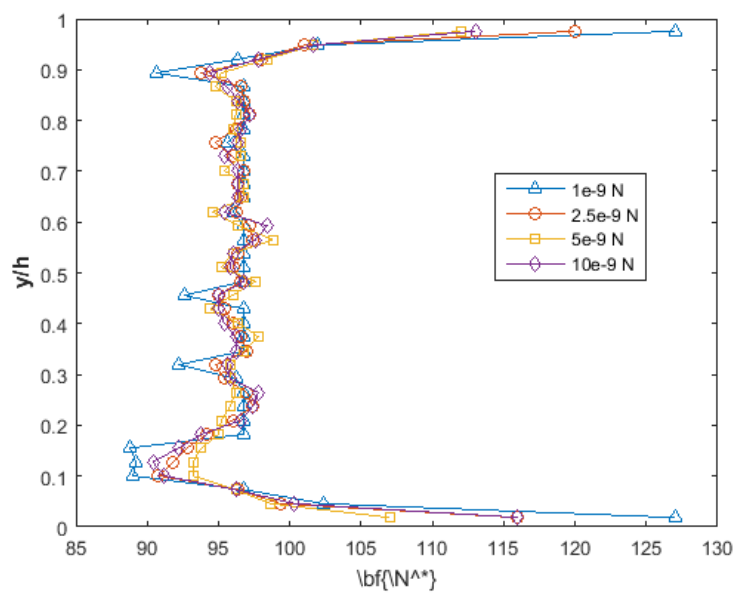

Figure 3. Poiseuille flow: Particle number density profile across the y-direction for different magnitudes of $F_{\text {ext }}$

At first, there seems to be no effect of the external driving forces on the shape of the profiles, as has been also shown in respective atomic-scale simulations (Spetsiotis et al., 2018). We also observe significant ordering of fluid atoms at layers close to the walls and we attribute this 
fact to the SPH technique behavior at the boundaries, where interactions of wall with fluid atoms exist. This result comes from Eq. (13), which has the advantage of conserving the mass exactly, but it shows edge effects for particles at the interface between the two different materials, i.e., solid wall - fluid. In this way, while there exist discontinuities across the channel y-dimension, the average particle number density remains constant.

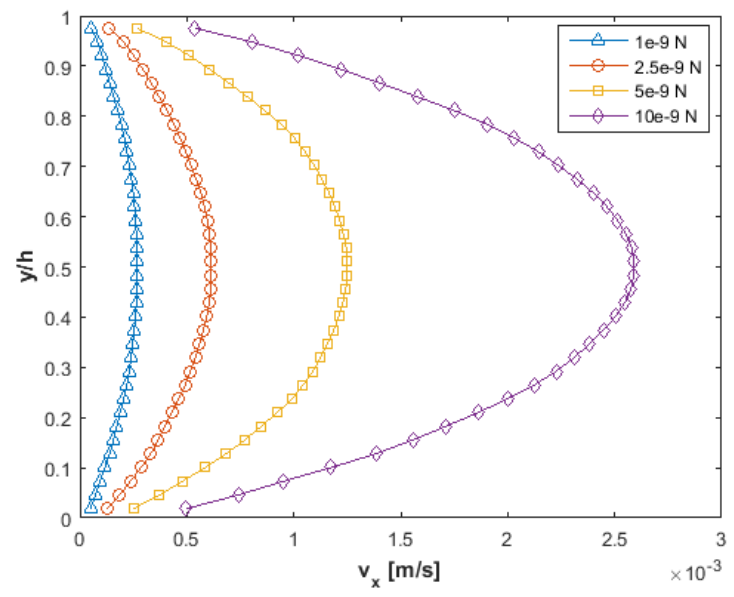

Figure 4. Poiseuille flow: $x$-Velocity profile across the $y$-direction for different magnitudes of $F_{\text {ext. }}$. Lines are $2^{\text {nd }}$ order parabolic fits

Moreover, we investigate the impact of the magnitude of the external applied force on fluid velocity (Figure 4). The velocity profiles seem to fit well on parabolas. Greater magnitude of $F_{\text {ext }}$ leads to greater velocity values, as expected from the continuum theory. It is of interest to note that a velocity bias is observed near the wall, representing the existence of slip velocities at the boundaries. We attribute this effect to the SPH technique behavior at the boundaries which can generate unrealistic effects. In Gomez-Gesteira (2010), it is noted that the velocity fall to zero when we approach the boundaries, while the density does not. Creation of realistic boundary conditions is still an open topic in SPH methods.

\subsection{Water tank drainage}

In this example we present the drainage of a rectangular tank with a hole in the lower right wall. For the ease of simulation, the model is considered $2 \mathrm{D}$, as a $(x, y)=\left(1.05 \times 10^{-3} \mathrm{~m}, 1.05 \times 10^{-3} \mathrm{~m}\right)$ non-periodic simulation box. Computational cost is minimal at this dimension scale, although larger scale problems can be tackled without any significant change in our SPH software. Water particles fill the tank and exit through a hole strip in the lower part of the right wall when simulation starts due to gravity. Simulation runs for $3 \times 10^{4}$ timesteps, where the timestep $\Delta t=2 \times 10^{-5} \mathrm{sec}$, corresponding to a real simulation time of $0.6 \mathrm{sec}$ and seems adequate to reveal the full evolution of the phenomenon (Figure 5). Drainage time has been found short and we attribute this to the small system dimension.

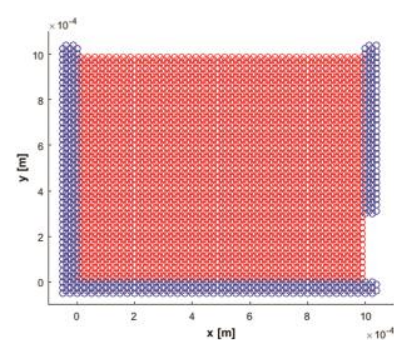

$\mathrm{t}=0 \mathrm{sec}$

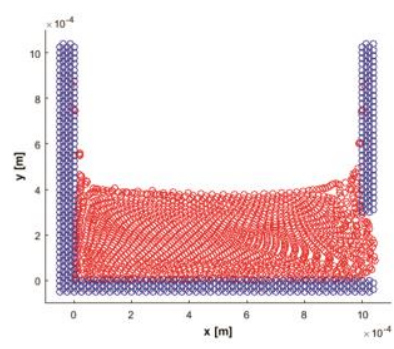

$\mathrm{t}=0.032 \mathrm{sec}$

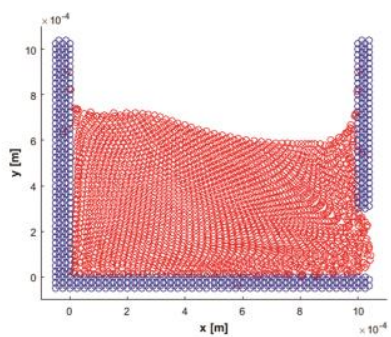

$\mathrm{t}=0.016 \mathrm{sec}$

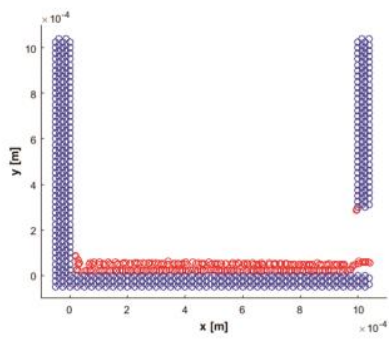

$\mathrm{t}=0.060 \mathrm{sec}$
Figure 5. Water tank drainage

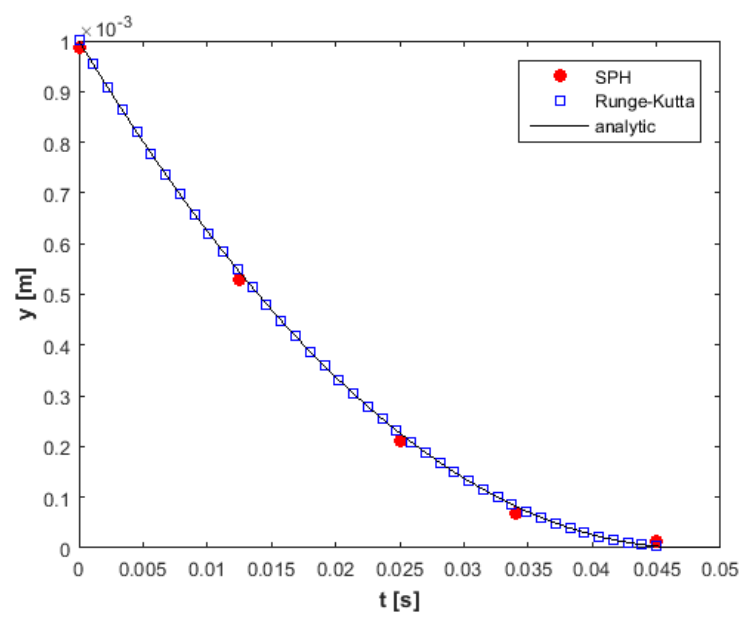

Figure 6. Comparison of the SPH, Runge-Kutta and analytical methods for the water tank drainage problem

To partially check the validity of the SPH result, comparison is made with the well-known analytical and numerical results. The draining of a tank with a hole is a problem, first described by Torricelli. If $S$ is the area ratio ("strip"/tank cross section) and $g$ the acceleration due to gravity, then water emerges from the hole with velocity $u_{h}=\sqrt{2 g y}$ and the variation of fluid height in time is given by the ordinary differential equation

$$
\frac{d y}{d t}=-s \sqrt{2 g} \sqrt{y}
$$

The analytical solution of Eq. (20) can be easily found as

$$
y(t)=\left(y_{0}^{1 / 2}-\frac{s \sqrt{2 g}}{2} t\right)^{2}
$$

where $y_{0}$ the initial height of water inside the tank. 
If we plot the numerical solution by a fourth/fifth order Runge-Kutta method of Eq. (20), the analytical solution, Eq. (21), and the variation of fluid average depth obtained by the SPH method, we obtain the results of Figure 6 . Our $\mathrm{SPH}$ results seem to fit very well to the model of Eqs. (20) and (21).

\section{Conclusions}

We have presented a smoothed particle hydrodynamics (SPH) numerical model and simulation results of water in three different examples: Couette and Poiseuille flows and the water tank drainage problem. We have shown that SPH, as a purely particle method, has many similarities to Molecular Dynamics, the well-documented atomistic simulation method, as well as to mesoscopic methods such as Dissipative Particle Dynamics. However, to address practical engineering problems in these fields we have to reduce the required CPU time for SPH and more generally for particle methods compared to classical CFD methods, especially when working with well-suited software platforms, such as Large-scale Atomic/Molecular Massively Parallel Simulator.

It should be mentioned that the method seems to reproduce well most macroscopic problems but fails to incorporate nano- and micro-scale effects, such as the wall effect on fluid properties and transport properties. We also have pointed out unrealistic effects in fluid flows close to the wall boundaries. Development of realistic boundary conditions is still an open topic in SPH methods. Further research on the applicability of the method in CFD and Hydraulics problems, would point out the scale limits and try to bridge with atomic-scale methods so as to suggest a hybrid multiscale modeling system.

\section{References}

Bian X., Li Z. and Karniadakis G.E. (2015), Multi-resolution flow simulations by smoothed particle hydrodynamics via domain decomposition, Journal of Computational Physics, 297, 131155.

Bouscasse B., Calagrossi A., Marrone S. and Antuono M. (2013), Non linear water interaction with floating bodies in $\mathrm{SPH}$, Journal of Fluids and Structures, 42, 112-129.

Eral H.B., D. van den Ende, Mugele F. and Duits M.H.G. (2009), Influence of confinement by smooth and rough walls on particle dynamics in dense hard-sphere suspensions, Physical Review E, 80, 061403.

Gingold R.A. and Monaghan J.J. (1977), Smoothed particle hydrodynamics: theory and application to non-spherical stars, Monthly Notices of the Royal Astronomical Society, 181, 375-389.

Gomez-Gesteira M., Rogers B.D., Dalrymple R.A. and Crespo A.J.C. (2010), State-of-the-art of classical SPH for free-surface flows, Journal of Hydraulic Research, 48, 6-27.

Hérault A., Bilotta G. and Dalrymple A.R (2010), SPH on GPU with CUDA, Journal of Hydraulic Research, 48, 74-79.

Hu X.Y. and Adams N.A. (2006), A multi-phase SPH method for macroscopic and mesoscopic flows, Journal of Computational Physics, 213, 844-861.

Koumoutsakos P. (2005), Multiscale flow simulations using particles, Annual Review of Fluid Mechanics, 37, 457-487.
Lei H., Fedosov D.A. and Karniadakis G.E. (2011), Timedependent and outflow boundary conditions for Dissipative Particle Dynamics, Journal of Computational Physics, 230, 3765-3779.

Li Z., Yazdani A., Tartakovsky A. and Karniadakis G.E. (2015), Transport dissipative particle dynamics model for mesoscopic advection-diffusion-reaction problems, The Journal of Chemical Physics, 143, 014101.

Liakopoulos A. and Sofos F. (2016), Computational Fluid Dynamics with MatLab, Lecture Notes, Department of Civil Engineering, University of Thessaly.

Liakopoulos A., Sofos F. and Karakasidis T.E. (2016), Friction factor in nanochannel flows, Microfluidics and Nanofluidics, 20, 1-7.

Liakopoulos A., Sofos F. and Karakasidis T.E. (2018), Modelling Environmental Flows with Lagrangian Particle Methods, in: $14^{\text {th }}$ International Conference on Protection and Restoration of the Environment, Thessaloniki, GR.

Liu M.B. and Liu G.R. (2010), Smoothed Particle Hydrodynamics (SPH): an Overview and Recent Developments, Archives of Computational Methods in Engineering, 17, 25-76.

Lykov K., Li X., Lei H., Pivkin I.V. and Karniadakis G.E. (2015), Inflow/Outflow Boundary Conditions for Particle-Based Blood Flow Simulations: Application to Arterial Bifurcations and Trees, PLoS Computional Biology, 11, 1-13.

Monaghan J.J. (1988), An introduction to SPH, Computer Physics Communications, 48, 89-96.

Monaghan J.J. (1992), Smoothed Particle Hydrodynamics, Annual Review of Astronomy and Astrophysics, 30, 543-574.

Pivkin I.V. and Karniadakis G.E. (2005), A new method to impose no-slip boundary conditions in dissipative particle dynamics, Journal of Computational Physics, 207, 114-128.

Plimpton S. (1995), Fast Parallel Algorithms for Short-Range Molecular Dynamics, Journal of Computational Physics, 117, 1-19.

Shao S. (2009), Incompressible SPH simulation of water entry of a free-falling object, International Journal for Numerical Methods in Fluids, 59, 91-115.

Silling S.A. and Lehoucq R.B. (2010), Peridynamic theory of solid mechanics, Advances in Applied Mechanics, 44, 73-168.

Song B., Pazouki A. and Pöschel T. (2018), Instability of SPH applied to Poiseuille flow, Computers \& Mathematics with Applications, 76, 1447-1457.

Sofos F., Karakasidis T.E. and Liakopoulos A. (2009), Transport properties of liquid argon in krypton nanochannels: Anisotropy and non-homogeneity introduced by the solid walls, International Journal of Heat and Mass Transfer, 52, 735-743.

Sofos F., Karakasidis T.E. and Liakopoulos A. (2013), Parameters affecting slip length at the nanoscale, Journal of Computational \& Theoretical Nanoscience, 10, 1-3.

Sparreboom W., van den Berg A. and Eijkel J.C.T (2009), Principles and applications of nanofluidic transport, Nature Nanotechnology, 4, 713-720.

Spetsiotis D., Sofos F., Karakasidis T.E., Kasiteropoulou D. and Liakopoulos A. (2018), Multi-parameter analysis of water flows in nanochannels, Desalination and Water Treatment, 125, 8-15.

Stukowski A. (2010), Visualization and analysis of atomistic simulation data with OVITO - the Open Visualization Tool, Modelling and Simulation in Material Science Engineering, 18, 015012.

White F.M. (1991), Viscous Fluid Flow, Second Edition, McGrawHill, New York. 